\title{
Prevalence of anti-hepatitis $B$ surface antibodies among children and adolescents vaccinated in infancy and effect of booster dose administered within a pilot study
}

\author{
R. BASSAL ${ }^{1} \dagger^{*}$, M. P. MARKOVICH ${ }^{1,2} \dagger, M$. WEIL $^{3}$, E. SHINAR ${ }^{4}$, Y. CARMELI $^{5}$,

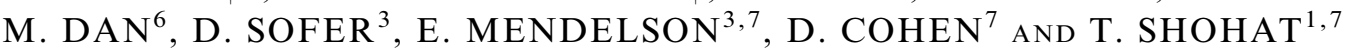 \\ ${ }^{1}$ Israel Center for Disease Control, Ministry of Health, Gertner Institute for Health Policy Research, Chaim \\ Sheba Medical Center, Tel Hashomer, Ramat-Gan, Israel \\ ${ }^{2}$ Israeli Veterinary Services, P.O. Box 12, Bet Dagan, Israel \\ ${ }^{3}$ Central Virology Laboratory, Ministry of Health, Public Health Services, Chaim Sheba Medical Center, \\ Tel Hashomer, Ramat-Gan, Israel \\ ${ }^{4}$ Magen David Adom, Ramat-Gan, Israel \\ ${ }^{5}$ Division of Epidemiology, Tel Aviv Sourasky Medical Center, Tel Aviv, Israel \\ ${ }^{6}$ Infectious Diseases Unit, Wolfson Medical Center, Holon, Israel \\ ${ }^{7}$ School of Public Health, Sackler Faculty of Medicine, Tel-Aviv University, Tel-Aviv, Israel
}

Received 8 March 2017; Final revision 21 August 2017; Accepted 21 August 2017; first published online 14 September 2017

\section{SUMMARY}

We determined the prevalence of anti-hepatitis B surface antibodies (anti-HBs) among children and adolescents vaccinated for hepatitis B virus in infancy as part of the routine vaccination programme. A representative serum sample of the Israeli population age 0-19 was tested. In a separate pilot study, a booster dose of hepatitis B vaccine was administered to 31 candidates for national service, who were fully vaccinated in infancy and tested negative for hepatitis B surface antibodies at age 17-19 years and anti-HBs antibodies were assessed 8 weeks later. Of the 1273 samples tested, $631(49 \cdot 6 \%)$ were positive to anti-HBs antibodies. Seropositivity rates were $89 \cdot 5 \%$ among infants aged 6-12 months and declined significantly with age to $20 \cdot 7 \%$ at age 19 years. No differences in seropositivity rates were observed between Jews and Arabs, males and females and those born in Israel and in other countries. Seroconversion rate among the 31 individuals who received a booster dose was 90·3\% (95\% CI: 75·1-96.6\%). We recommend a booster dose for healthcare personnel before starting to work at the health care facility.

Key words: Hepatitis B, Israel, seroprevalence, vaccination.

\section{INTRODUCTION}

Infection with hepatitis B virus (HBV) can cause acute and chronic liver diseases [1, 2]. The virus is transmitted by parenteral or mucosal exposure to infected blood and serous fluids [2, 3]. In highly endemic

\footnotetext{
* Author for correspondence: Ravit Bassal Ph.D. Head of the Laboratory Unit, Israel Center for Disease Control, Chaim Sheba Medical Center, Tel-Hashomer 52621, Israel. (E-mail: ravit.bassal@, moh.health.gov.il)

$\dagger$ These authors contributed equally to this work.
}

areas, HBV is most commonly spread from mother to child at birth or through horizontal transmission during the first 5 years of life [3]. Sexual transmission of hepatitis B may occur, particularly in men who have sex with men and heterosexual persons with multiple sex partners or contact with sex workers $[3,4]$. Transmission of the virus may also occur through reuse of needles and syringes [3, 4].

It is estimated that 240 million people are chronically infected with hepatitis B and more than 686000 people around the world die annually due to 
complications of hepatitis $\mathrm{B}$, including cirrhosis and liver cancer [3-5].

In 1992, the second generation recombinant DNA hepatitis B vaccine was introduced to the routine immunization programme in Israel and is given since in three doses, at birth, 1 and 6 months of age $[6,7]$.

Although data on vaccine uptake in Israel are highly available, a few national sero-surveys assessed the seroprevalence of anti-HBs antibodies among the Israeli population following the introduction of HBV vaccine [8-10].

The aim of the present study was to determine the seropositivity rate of anti-HBs antibodies among children and adolescents aged $0-19$ years, 19 years following the introduction of the vaccine. In a separate pilot study, we evaluated the seroconversion rate of a group of 17-19 years old adolescents who tested negative to anti-HBs antibodies after the administration of a single dose of $\mathrm{HBV}$ vaccine.

\section{METHODS}

\section{Study design}

We conducted a cross-sectional sero-survey using the Israel national serum bank established in 1997 by the Israel Center for Disease Control. Serum samples from all regions of Israel are collected throughout the year. Serum samples of persons aged $<18$ years and $>55$ years are residual samples from diagnostic laboratories, while samples of persons aged 19-55 are collected from both healthy blood donors and residual serum samples from diagnostic laboratories. All 1273 samples collected in 2011 were used for the current study. For each sample, data retrieved were sampling date, date of birth, gender, place of residence, country of birth and ethnic group.

\section{Sampling}

Inclusion criteria for the seroprevalence study were all samples collected between January and June 2011 from children and adolescents aged 0-19 years, born after 1 January 1992, the date the vaccine was introduced to the routine immunization programme in Israel. The samples were stratified by gender, the following age groups: $0.00-0.49, \quad 0.50-1.99,2 \cdot 00-4.99, \quad 5 \cdot 00-9.99$, $10.00-14.99$ and $15 \cdot 00-19.99$ years and by ethnicity. In total, 170 samples were required to detect a difference of $15 \%$ or higher in seropositivity rates between age groups with power $(1-\beta)$ of $80 \%$ and $\alpha$ of $0 \cdot 05$.
Table 1. Socio-demographic characteristics of the study population

\begin{tabular}{lll}
\hline \hline & $\mathrm{N}$ & $\%$ \\
\hline Age (years) & & \\
$0.00-0 \cdot 49$ & 29 & $2 \cdot 3$ \\
$0 \cdot 50-1 \cdot 99$ & 128 & $10 \cdot 0$ \\
$2 \cdot 00-4 \cdot 99$ & 229 & $18 \cdot 0$ \\
$5 \cdot 00-9 \cdot 99$ & 257 & $20 \cdot 2$ \\
$10 \cdot 00-14 \cdot 99$ & 276 & $21 \cdot 7$ \\
$15 \cdot 00-19 \cdot 99$ & 354 & $27 \cdot 8$ \\
Gender & & \\
Male & 699 & $54 \cdot 9$ \\
Female & 574 & $45 \cdot 1$ \\
Ethnic group & & \\
Jew & 882 & $69 \cdot 3$ \\
Arab & 391 & $30 \cdot 7$ \\
Birth country & & \\
Israel & 1219 & $95 \cdot 8$ \\
Other & 53 & \\
\hline \hline
\end{tabular}

\section{Booster response evaluation}

In a framework of a pilot study, we approached candidates for voluntary national service at the National Blood Services, born after 1 January 1992. These candidates were required to present certificate of hepatitis B vaccination, and an updated anti hepatitis B antibodies level test result as part of the recruitment process. Those who received a full immunization course in infancy and tested negative for anti-HBs antibodies received a booster dose and provided test results for anti-HBs antibodies within 8 weeks after vaccination. A sample size of 31-52 samples was sufficient to demonstrate $98 \%$ seropositivity (95\% CI: 95-99\%) after receiving a booster dose.

\section{Laboratory testing}

Anti-HBs antibodies level was determined for each sample using the Vitek Immuno Diagnostic Assay System (VIDAS) Anti-Hbs Total Quick kit, according to the manufacturer's instructions and were expressed in $\mathrm{mIU} / \mathrm{ml} . \mathrm{IgG} \geqslant 12 \mathrm{mIU} / \mathrm{ml}$ was considered positive, $8 \leqslant \mathrm{IgG}<12 \mathrm{mIU} / \mathrm{ml}$ intermediate and $\mathrm{IgG}<8 \mathrm{mIU} / \mathrm{ml}$ was considered negative. Intermediate samples were considered negative for calculating seropositivity rate. Anti-HBc antibodies level was determined using the VIDAS anti-HBc Total II kit, bioMerieux, according to the manufacturer's instructions and were expressed in $\mathrm{mIU} / \mathrm{ml}$. IgG $<1 \mathrm{mIU} / \mathrm{ml}$ was considered negative, $1.0 \leqslant \operatorname{IgG}<1.4 \mathrm{mIU} / \mathrm{ml}$ intermediate and $\operatorname{IgG} \geqslant 1.4$ $\mathrm{mIU} / \mathrm{ml}$ was considered positive to Anti-HBc. The 


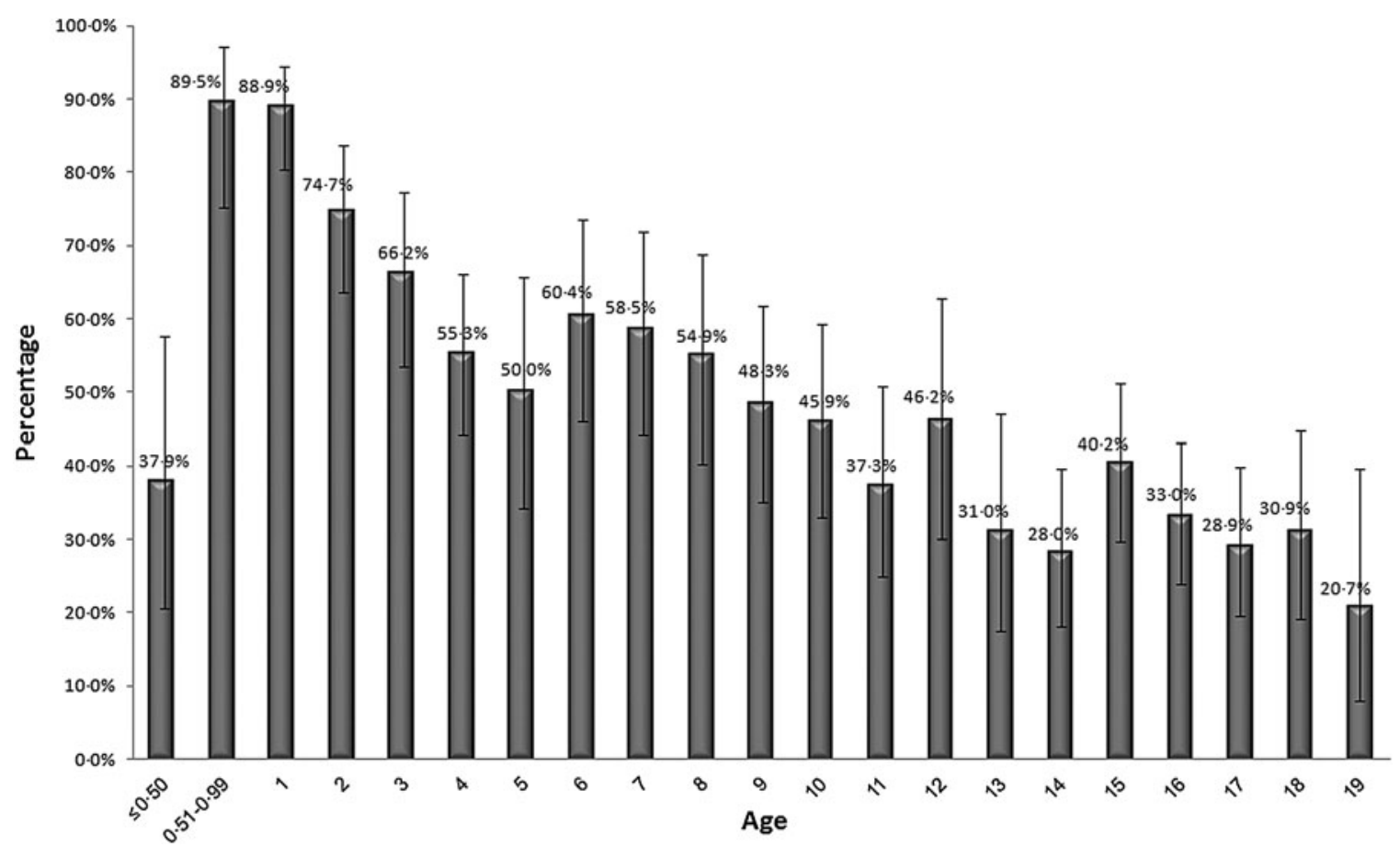

Fig. 1. Prevalence of anti-HBs antibodies by age.

Table 2. Association of seropositivity with sociodemographic characteristics of the study population

\begin{tabular}{lllll}
\hline \hline & Positive (\%) & OR & $95 \%$ CI & $P$-value \\
\hline Age (years) & & & & \\
$0 \cdot 50-1 \cdot 99$ & $114(89 \cdot 1)$ & Ref. & & \\
$2 \cdot 00-4.99$ & $149(65 \cdot 1)$ & $0 \cdot 23$ & $0 \cdot 12-0 \cdot 42$ & $<0 \cdot 01$ \\
$5 \cdot 00-9 \cdot 99$ & $140(54 \cdot 5)$ & $0 \cdot 15$ & $0 \cdot 08-0 \cdot 27$ & $<0 \cdot 01$ \\
$10 \cdot 00-14.99$ & $102(37 \cdot 0)$ & $0 \cdot 07$ & $0 \cdot 04-0 \cdot 13$ & $<0 \cdot 01$ \\
$15 \cdot 00-19 \cdot 99$ & $115(32 \cdot 5)$ & $0 \cdot 06$ & $0 \cdot 03-0 \cdot 11$ & $<0 \cdot 01$ \\
Gender & & & & \\
Male & $334(47 \cdot 8)$ & Ref. & & \\
Female & $297(51 \cdot 7)$ & $1 \cdot 17$ & $0.94-1 \cdot 46$ & $0 \cdot 16$ \\
Ethnic group & & & & \\
Jew & $435(49 \cdot 3)$ & Ref. & & \\
Arab & $196(50 \cdot 1)$ & $1 \cdot 03$ & $0 \cdot 81-1 \cdot 31$ & $0 \cdot 79$ \\
Birth country & & & & \\
Israel & $599(49 \cdot 1)$ & Ref. & & \\
Other & $32(60 \cdot 4)$ & $1 \cdot 58$ & $0.90-2 \cdot 77$ & $0 \cdot 11$ \\
\hline \hline
\end{tabular}

samples were tested in the Central Virology Laboratory of the Israel Ministry of Health.

\section{Ethic statement}

The study was approved by the Institutional Review Board at Sheba Medical Center, Tel Aviv Sourasky Medical Center and Wolfson Medical Center. All participants signed an informed consent.

\section{Data analysis}

Seropositivity rates were calculated by age and ethnic group. Analysis of trend was done using the Joinpoint regression analysis applied by the Joinpoint software (the Surveillance Research Program of the US National Cancer Institute, Version 4.4.0) [11]. Differences in the prevalence of seropositivity between subgroups were evaluated using chi-square analyses. The logistic regression model was used to evaluate the association between demographic characteristics and seropositivity, and to calculate odds ratios and 95\% confidence interval (CI). Statistical significance was determined using 2-sided test with an alpha level of 0.05. All analyses were performed using SAS software package (version 9.1.3, SAS Institute Inc., Cary, NC, USA).

\section{RESULTS}

A total of 1273 samples were tested. Table 1 presents the demographic characteristics of the study population. Of the samples tested, $699(54.9 \%)$ were males, $882(69 \cdot 3 \%)$ were Jews and $1219(95 \cdot 8 \%)$ were born in Israel. Totally, $631(49 \cdot 6 \%)$ samples tested positive for anti-HBs. Seropositivity rate was $37.9 \%$ up to age 6 months, followed by a peak at age 6-12 months (Fig. 1). As time elapsed since vaccination in infancy, 
Table 3. Seroprevalence of anti-Hepatitis B antibodies in different countries at different time periods after vaccination

\begin{tabular}{lllll}
\hline \hline Country & Authors & Number of years after vaccination & Seropositivity rate $(\%)$ & Reference \\
\hline Israel & Bassal et al. & 10 & $45 \cdot 9$ & Present study \\
China & But et al. & 10 & $81 \cdot 8$ & 16 \\
Italy & Zanetti et al. & 10 & $64 \cdot 0$ & 17 \\
Iran & Norouzirad et al. & 18 & $48 \cdot 9$ & 18 \\
Israel & Bassal et al. & 19 & $20 \cdot 7$ & Present study \\
China & But et al. & 19 & $68 \cdot 2$ & 16 \\
Egypt & El Mazahi et al. & 19 & $60 \cdot 0$ & 19 \\
Thailand & Chinchai et al. & 19 & $58 \cdot 6$ & 20 \\
Iran & Saffar et al. & 19 & $43 \cdot 9$ & 21 \\
Saudi Arabia & Alfaleh et al. & 19 & 38.0 & 22 \\
Palestinian Authority & Qawasmi et al. & 19 & $37 \cdot 6$ & 23 \\
USA & Middleman et al. & 19 & $24 \cdot 1$ & 24 \\
\hline \hline
\end{tabular}

seropositivity rates declined significantly $(P$-value $<$ $0 \cdot 01$ ) reaching $20 \cdot 7 \%$ at age 19 (Annual Percentage Change $=-5 \cdot 7$ ). Table 2 demonstrates the seropositivity rate by demographic characteristics of the study population. No differences in seropositivity rates were observed between Jews and Arabs $(P$-value $=0.79)$, males and females $(P$-value $=0 \cdot 16)$ and those born in Israel and others $(P$-value $=0 \cdot 11)$. Age was the only factor significantly associated with seropositivity rate.

In total, 553 samples which were positive to antiHBs were tested for anti-HBc. In 78 samples, there was not enough sera for further testing. Four samples $(1 \cdot 3 \%)$ were anti-HBc positive and $3(0 \cdot 5 \%)$ were intermediate.

Two hundred and sixteen candidates for voluntary national service, who presented documentation of hepatitis $\mathrm{B}$ vaccination in infancy, were approached. $139(64.4 \%)$ of them tested negative for anti-HBs antibodies, and 78 of them $(56 \cdot 1 \%)$ received a booster dose as part of the recruitment process. Thirty one (39.7\%) of them provided a second blood sample within 8 weeks since vaccination. The mean age and standard deviation of the 31 participants was $17 \cdot 6 \pm 0.6$ years, $93.3 \%$ were females and $89 \cdot 3 \%$ were born in Israel. Twenty-eight of 31 participants $(90 \cdot 3 \% ; 95 \% \mathrm{CI}$ : $75 \cdot 1-96 \cdot 6 \%$ ) tested positive for anti-HBs antibodies following vaccination. The three non-responders were all females, Jews, who were born in Israel.

\section{DISCUSSION}

In this cross-sectional study we have shown a continuous decline in seropositivity rate of anti-HBs antibodies with time since vaccination. Hepatitis B vaccine is administered to all newborns in Israel in the first $24 \mathrm{~h}$ following birth, at 1 month and 6 months.

Vaccination coverage for the third dose was 92 99\% between 1992 and 2015 [12, 13]. Taking into account the possibility of vaccine failure, estimated at $3 \%$ [14], it is expected that the highest seropositivity rate would be around $90 \%$ at the age group 6-12 months following the administration of three doses; indeed the seroprevalence in this age group was $89.5 \%$. We had no information regarding the health status of the study population, and we assume they were healthy. It was shown that children with chronic diseases have lower seroprotective levels in diseased children compared with normal children [15].

Table 3 presents the results of several studies that examined seropositivity rate 10 years and longer after vaccination. In the present study seropositivity rate 10 years following vaccination, was $45.9 \%$, lower compared with reports from other countries (Table 3) [16, 17]. Nineteen years following vaccination, seropositivity was $20 \cdot 7 \%$, lower than reported by China, Egypt, Thailand, Iran, Saudi-Arabia and The Palestinian Authority (Table 3) [16, 18-23] and similar to the USA [24]. A previous study performed in Israel among male recruits born after 1 January, 1992, evaluated seropositivity rate 18-20 years after vaccination have shown that only $33 \cdot 7 \%$ of male recruits were anti-HBs positive [8]. The observed differences in seropositivity rates between different studies could be explained by differences in vaccine programmes schedule, type of vaccine (plasma based vs. recombinant) and the number of doses administered.

The decline in seropositivity with time since vaccination raised the question of the need for a booster dose. Similar to earlier studies [24-26], seropositivity rate 
following a booster dose was very high $(90 \cdot 3 \%)$. Spada et al. demonstrated 98\% seroconversion 17 years after vaccination [25], Middleman et al. demonstrated 92\% seroconversion 16-19 years after vaccination [24] and Bagheri-Jamebozorgi et al. demonstrated 90.6\% seroconversion 20 years after vaccination [26].

Our results were higher than those reported by Hartal, who vaccinated male recruits, 18-20 years after initial vaccination with one dose demonstrated $81.5 \%$ seroconversion [8] and Chaves who challenged the participants 15 years after initial vaccination and demonstrated $71.4 \%$ seroconversion [27]. Although the high seropositivity rate observed among the revaccinated population, it should be kept in mind that $9 \cdot 7 \%$ were still negative.

We have demonstrated low rate $(1 \cdot 3 \%)$ of anti-HBc positive samples among those who were anti-HBs positive. Our results are in accordance with previous studies demonstrated hepatitis B breakthrough despite universal vaccination. Saffar et al. reported that $2 \cdot 5-3 \cdot 5 \%$ of 510 blood samples tested were anti-HBc positive [21] while Spada et al. in Italy [25] and Alfaleh in Saudi Arabia have shown no samples with anti-HBc positivity [22].

There is still controversy regarding the necessity of a booster dose implementation for risk groups $[20,28]$. Since health care workers, especially surgeons and those engaged in interventional procedures, are at higher risk of various blood-borne infections, including $\mathrm{HBV}$, and high vaccination coverage is needed, we recommend a booster dose for healthcare personnel before starting to work at the health care facility as described [29].

There are several limitations to the present study. (1) Although vaccination rates for HBV are high, we had no information regarding the vaccination history on each sample tested. (2) Since antibody testing after the administration of a booster dose was done within 8 weeks following vaccination, we cannot differentiate whether we observed a booster response or a primary response.

\section{ACKNOWLEDGEMENTS}

The authors would like to thank Nadia Pekurovski from the Israel Center for Disease Control, for her assistance in data collection and laboratory assistant and to Dr Veronica Gemdelman and Mrs. Hana Raz from MDA blood services for the blood samples collection. This research received no specific grant from any funding agency, commercial or not-for-profit sectors.

\section{DECLARATION OF INTEREST}

None.

\section{REFERENCES}

1. Liang TJ. Hepatitis B: the virus and disease. Hepatology 2009; 49: S13-S21.

2. Centers for Disease Control and Prevention (CDC). Hepatitis $B$ in: Epidemiology and Prevention of Vaccine-Preventable Diseases, The Pink Book. 13th edn. Atlanta, GA: US Department of Health and Human Services, Public Health Foundation, 2015. Chapter 10. 149-173. (https://www.cdc.gov/vaccines/pubs/pinkbook/ downloads/hepb.pdf). Accessed 5 March 2017.

3. World Health Organization (WHO). Media centre: hepatitis B. July 2016. (http://www.who.int/mediacentre/factsheets/fs204/en/). Accessed 5 March 2017.

4. Centers for Disease and Control (CDC). Hepatitis B FAQs for Health Professionals (http://www.cdc.gov/ hepatitis/hbv/hbvfaq.htm). Accessed 5 March 2017.

5. Lozano R, et al. Global and regional mortality from 235 causes of death for 20 age groups in 1990 and 2010: a systematic analysis for the global burden of disease study 2010. Lancet 2012; 380: 2095-2128.

6. Tesher D, Somekh E. Hepatitis B vaccines and the immune response to them. Israeli Journal of Pediatrics 2010;73: 4.

7. Israeli Division of Epidemiology, Public Health Services Ministry of Health. Hepatitis B in: Vaccines Book. Updates: August 2016.

8. Hartal M, et al. Seroprevalence of anti-HBs antibodies at young adulthood, before and after a booster vaccine dose, among medical personnel vaccinated in infancy. Vaccine 2015; 33: 4878-4885.

9. Gold Y, et al. Decreased immune response to hepatitis B eight years after routine vaccination in Israel. Acta Paediatrica 2003; 92: 1158-1162.

10. Michaiel R, et al. Vertical HBV transmission in Jerusalem in the vaccine era. Harefuah 2012; 151: 671-674, 721, 722.

11. Joinpoint. Statistical Research and Applications Branch, National Cancer Institute. Joinpoint Regression Program, Version 4.4.0. (http://surveillance.cancer.gov/joinpoint). Accessed 5 January 2017.

12. Central Bureau of Statistics. Children immunized out of those registered in the mother and infant centers. Table 6.12 Published 10 Septemaber 2015.

13. World Health Organization (WHO). WHO vaccinepreventable diseases: monitoring system, Israel. Updated December 2016. (http://apps.who.int/immunization_monitoring/globalsummary/coverages?c=ISR). Accessed 5 March 2017.

14. Rots NY, et al. Hepatitis B vaccination strategies tailored to different endemicity levels: some considerations. Vaccine 2010; 28: 893-900.

15. El-Asheer OM, et al. Immunogenicity of recombinant hepatitis B vaccine among routinely vaccinated healthy and chronically Ill children in Assiut, upper Egypt. Gastroenterology Research 2015; 8: 222-227. 
16. But DY, et al. Twenty-two years follow-up of a prospective randomized trial of hepatitis B vaccines without booster dose in children: final report. Vaccine 2008; 26: 6587-6591.

17. Zanetti AR, et al. Long-term immunogenicity of hepatitis B vaccination and policy for booster: an Italian multicentre study. Lancet 2005; 366: 1379-1384.

18. Norouzirad R, et al. Serum levels of anti-hepatitis B surface antibody among vaccinated population aged 1 to 18 years in Ahvaz city southwest of Iran. Hepatitis Monthly 2014; 14: e13625.

19. El Mazahi MM, et al. Long term immunity to hepatitis B vaccine among a sample of secondary school students in Damietta. Journal of American Science 2014; 10: 140-145.

20. Chinchai T, et al. Long-term humoral and cellular immune response to hepatitis B vaccine in high-risk children 18-20 years after neonatal immunization. Viral Immunology 2009; 22: 125-130.

21. Saffar H, et al. Prevalence of hepatitis B virus seromarkers in young adults vaccinated at birth; impact on the epidemiology of hepatitis B infection in Iran. Hepatitis Monthly 2014; 14: e17263.

22. Alfaleh F, et al. Long-term protection of hepatitis B vaccine 18 years after vaccination. Journal of Infection 2008; 57: 404-409.

23. Qawasmi M, et al. Age-dependent decrease of anti-HBs titers and effect of booster doses using two different vaccines in Palestinian children vaccinated in early childhood. Human Vaccines \& Immunotherapeutics 2015; 11: 1717-1724.

24. Middleman AB, et al. Duration of protection after infant hepatitis B vaccination series. Pediatrics 2014; 133: e1500-e1507.

25. Spada E, et al. Hepatitis B immunity in teenagers vaccinated as infants: an Italian 17-year follow-up study. Clinical Microbiology and Infection 2014; 20: O680 O686.

26. Bagheri-Jamebozorgi M, et al. The persistence of antiHBs antibody and anamnestic response 20 years after primary vaccination with recombinant hepatitis $\mathrm{B}$ vaccine at infancy. Human Vaccines and Immunotherapeutics 2014; 10: 3731-3736.

27. Chaves SS, et al. Persistence of long-term immunity to hepatitis B among adolescents immunized at birth. Vaccine 2012; 30: 1644-1649.

28. Lu CY, et al. Waning immunity to plasma-derived hepatitis B vaccine and the need for boosters 15 years after neonatal vaccination. Hepatology 2004; 40: 1415-1420.

29. Israeli Ministry of Health. Director general's circular of 08/2016: vaccination of healthcare students and health system employees. September 2016. (http://www. health.gov.il/English/Topics/Vaccination/Pages/health_ students_vaccines.aspx). Accessed 5 March 2017. 